\title{
RECENT PROGRESS ON THE TATE CONJECTURE
}

\author{
BURT TOTARO
}

\begin{abstract}
We survey the history of the Tate conjecture on algebraic cycles. The conjecture is closely related with other big problems in arithmetic and algebraic geometry, including the Hodge and Birch-Swinnerton-Dyer conjectures. We conclude by discussing the recent proof of the Tate conjecture for K3 surfaces over finite fields.
\end{abstract}

\section{Contents}

1. Origins of the Tate conjecture, 1962-1965

2. The broader context: Variants of the Tate conjecture

3. First results over finite fields: The Tate conjecture for divisors on abelian varieties

4. Main tool: The Kuga-Satake correspondence

5. Further results over finite fields: The Tate conjecture for K3 surfaces

6. Conclusion

Acknowledgments

The Tate conjecture is a central problem in arithmetic geometry. Roughly speaking, the conjecture predicts a description of all subvarieties of a given algebraic variety over a field $k$ in terms of the representation of the Galois group of $k$ on étale cohomology. (For $k$ a finite field, computing this Galois representation is essentially elementary, amounting to counting points of the variety over a few finite extensions of $k$.) So the Tate conjecture would make much of algebraic geometry and number theory accessible to computation.

More broadly, the Tate conjecture is closely intertwined with several of the other central conjectures of number theory and algebraic geometry, including the Hodge conjecture and the Birch-Swinnerton-Dyer conjecture. Even if the Tate conjecture eventually turns out to be false (which I do not expect), it has more than justified itself by the results and connections it has inspired.

Metaphorically, one can think of this family of conjectures as aiming to describe all the solutions to a polynomial equation, or equivalently describing all the rational points of an algebraic variety over a given field. The conjectures connect this problem to computable problems in topology. In some special cases, such as a

Received by the editors May 30, 2017.

2010 Mathematics Subject Classification. Primary 14C25; Secondary 14F20, 14G15, 14J28.

This work was supported by NSF grant DMS-1303105. 
surface mapping to a curve with generic fiber an elliptic curve, the Tate conjecture would literally describe all the rational points of the generic fiber.

This paper recalls the history of the conjecture, including the influences on Tate and his proof of the conjecture in some important cases. We discuss progress over several decades by Artin, Swinnerton-Dyer, Nygaard, Ogus, Tankeev, André, Moonen, and others. We conclude with a striking recent advance, the proof of the Tate conjecture for K3 surfaces over finite fields, by Charles, Kim, Madapusi Pera, and Maulik. These proofs extend the long-standing connections between the Tate conjecture and finiteness problems in arithmetic geometry. The methods raise the hope of progress for other varieties, some of which has already begun.

The account of recent work given here is far from complete, and many related results could not be included. Some surveys with additional material are Tate's own "Conjectures on algebraic cycles in I-adic cohomology" 45, Milne's "The Tate conjecture over finite fields" 31], and Benoist's Séminaire Bourbaki talk on the Tate conjecture for K3 surfaces [4].

\section{Origins of the Tate Conjecture, 1962-1965}

Here we state the Tate conjecture and discuss its early history, including several related conjectures which were proposed around the same time.

The Tate conjecture (published in 1965 [42]) was inconceivable until the definition of étale cohomology by Grothendieck and his collaborators in the early 1960s. Étale cohomology integrated algebraic geometry and topology in a new way. In the 1950s, Serre had introduced cohomological methods into algebraic geometry over any field, but the resulting invariants did not behave like cohomology groups in topology. By contrast, étale cohomology gives l-adic cohomology groups for an algebraic variety $X$ over any field $k$ with many of the same properties as the singular cohomology of a complex algebraic variety. As a bonus, $l$-adic cohomology has extra structure: it is a representation of the absolute Galois group $\operatorname{Gal}\left(k_{s} / k\right)$ (the automorphism group of the separable closure $k_{s}$ over $k$ ).

The specific version of $l$-adic cohomology used in this paper, $H^{i}\left(X, \mathbf{Z}_{l}\right)$, means geometric étale cohomology $H_{\mathrm{et}}^{i}\left(X_{k_{s}}, \mathbf{Z}_{l}\right)$. Here $l$ denotes a prime number invertible in $k$, and $\mathbf{Z}_{l}$ is the ring of $l$-adic integers, the inverse limit of the rings $\mathbf{Z} / l^{r}$ as $r$ goes to infinity.

To give an example where this Galois representation has a simple interpretation, let $X$ be an abelian variety of dimension $g$ over a field $k$. Then the étale homology $H_{1}\left(X, \mathbf{Z}_{l}\right)$ is the Tate module of $X$ : the inverse limit of the $l$-power torsion subgroups of the abelian group $X\left(k_{s}\right)$. It is known that $H_{1}\left(X, \mathbf{Z}_{l}\right)$ is isomorphic to $\left(\mathbf{Z}_{l}\right)^{2 g}$, but the Galois action on this group is highly nontrivial, corresponding to the Galois action on torsion points of $X\left(k_{s}\right)$. That is, the nontriviality of the Galois action reflects the fact that the torsion points of $X$ over $k_{s}$ need not be defined over $k$. The étale cohomology $H^{1}\left(X, \mathbf{Z}_{l}\right)$ is the dual group, $\operatorname{Hom}_{\mathbf{Z}_{l}}\left(H_{1}\left(X, \mathbf{Z}_{l}\right), \mathbf{Z}_{l}\right)$.

A closed subvariety $Y$ of codimension $a$ in a smooth variety $X$ determines an element of $H^{2 a}\left(X, \mathbf{Z}_{l}(a)\right)$. Here $\mathbf{Z}_{l}(a)$ denotes the Tate twist. That is, $\mathbf{Z}_{l}(1)$ denotes the inverse limit of the roots of unity $\mu_{l^{r}}\left(k_{s}\right)$ as $r$ goes to infinity; so it is isomorphic to $\mathbf{Z}_{l}$, but with a nontrivial action of the Galois group of $k$. For any integer $a, \mathbf{Z}_{l}(a)$ denotes the $a$ th tensor power of $\mathbf{Z}_{l}(1)$. (For negative integers, $\mathbf{Z}_{l}(-a)$ means the dual of $\mathbf{Z}_{l}(a)$.) 
More precisely, the class of a subvariety $Y$ of $X$ lies in the subgroup of $H^{2 a}\left(X, \mathbf{Z}_{l}(a)\right)$ fixed by the Galois group of $k$, because we are considering subvarieties defined over $k$. The Tate conjecture asserts a converse:

Conjecture 1.1. Let $k$ be a finitely generated field over the prime field (that is, over $\mathbf{Q}$ or a finite field). Let $X$ be a smooth projective variety over $k$. Then the $\mathbf{Q}_{l}$-linear subspace of $H^{2 a}\left(X, \mathbf{Q}_{l}(a)\right)$ fixed by the Galois group of $k$ is spanned by the classes of codimension-a subvarieties of $X$.

Define an algebraic cycle on $X$ to be a finite linear combination of subvarieties of $X$. Then the Tate conjecture can be rephrased as saying that every element of $l$-adic cohomology fixed by the Galois group is the class of some algebraic cycle with $\mathbf{Q}_{l}$ coefficients. A cohomology class fixed by the Galois group is sometimes called a Tate class. Thus the conjecture says that every Tate class is algebraic.

The Tate conjecture depends on the fact that the base field $k$, a finitely generated field over the prime field, is small in some sense. The analogous statement fails when $k$ is algebraically closed (in which case the Galois representation is trivial), and also for some other fields such as $p$-adic fields. (An exception is the case of $p$-adic cohomology of varieties with totally degenerate reduction over a $p$-adic field, where Raskind conjectured the analog of the Tate conjecture [37.) The hope is that the Galois representation on $l$-adic cohomology carries maximal information for finitely generated fields, the fields of most arithmetic interest.

The Tate conjecture grew out of Tate's conjecture that the Tate-Shafarevich group $\amalg$ of an abelian variety over a global field should be finite; the precise relation between these conjectures is discussed below. (A global field means a number field or the function field of a curve over a finite field.) In fact, Tate's 1962 ICM paper stated the Tate conjecture for divisors (codimension-1 cycles) on a surface, as well as the conjecture that $\amalg$ is finite.

Tate explained in 1994: "If $\amalg$, or at least its $l$-primary part, were not finite, then the Galois cohomology of the abelian variety would be a mess, and the determination of the group of rational points by 'descent' would be ineffective" [4]. A related conjecture around the same time was the Birch-Swinnerton-Dyer conjecture, predicting that the rank of the Mordell-Weil group of an elliptic curve over a number field is equal to the order of vanishing of the $L$-function at $s=1$ [5]. More precisely, the leading coefficient of the $L$-function should be given by an explicit formula in terms of the order of $\amalg$.

The hope for the finiteness of $\amalg$ led Tate fairly naturally to the Tate conjecture for divisors. The Tate conjecture for higher-codimension cycles did not have such a clear motivation; beyond the elegance of the statement, Tate had only "some very meager evidence" from the case of Fermat hypersurfaces [45]. Tate wrote several letters to Serre in 1963 and 1964 about his path to the conjecture [9].

There is a rough analogy between the Tate conjecture and the Hodge conjecture. The Hodge conjecture would describe the classes of algebraic cycles in the rational cohomology of a smooth complex projective variety $X$ in terms of the Hodge structure on cohomology. Namely, there is a direct-sum decomposition of the cohomology of $X$ with complex coefficients:

$$
H^{r}(X, \mathbf{C})=\bigoplus_{a} H^{a}\left(X, \Omega^{r-a}\right)
$$


where $\Omega^{j}$ denotes the sheaf of regular $j$-forms on $X$. The dimensions $h^{r-a, a}$ of the complex vector spaces $H^{a}\left(X, \Omega^{r-a}\right)$ are traditionally summarized in the Hodge diamond, which for $X$ of dimension 2 looks like

$\begin{array}{ccccc} & & h^{2,2} & & \\ h^{0,2} & h^{1,2} & & h^{2,1} & \\ & & h^{1,1} & & h^{2,0} \\ & h^{0,1} & & h^{1,0} & \\ & & h^{0,0} . & & \end{array}$

The class of an algebraic cycle of codimension $a$ in $X$ lies in $H^{2 a}(X, \mathbf{Q})$ and also in the middle piece $H^{a}\left(X, \Omega^{a}\right)$ of the Hodge decomposition (the central column of the Hodge diamond). The Hodge conjecture predicts the converse: any element of $H^{2 a}(X, \mathbf{Q})$ whose image in $H^{2 a}(X, \mathbf{C})$ is in the middle piece of the Hodge decomposition must be the class of some algebraic cycle with $\mathbf{Q}$ coefficients 15, Definition 3.3.20]. In other words, every Hodge class should be algebraic. Or again: the group of algebraic classes should be determined by the Hodge structure of $X$, the combination of the rational structure $H^{2 a}(X, \mathbf{Q})$ inside $H^{2 a}(X, \mathbf{C})$ with the Hodge decomposition.

One big difference between the two conjectures is that the Hodge conjecture for divisors is known (the Lefschetz $(1,1)$-theorem [15, Proposition 3.3.2]), whereas the Tate conjecture for divisors is a major open problem.

Although there are no obvious implications between the Tate and Hodge conjectures, there have been implications for particular classes of varieties. For example, some cases of the Tate conjecture for divisors have been proved by showing that a Tate class lifts to a Hodge class, on a suitable lift of the given variety to characteristic 0 .

The case of divisors shows that the Tate conjecture is harder than the Hodge conjecture. Why should that be? Naively, one might feel that Galois representations are more "algebraic", and therefore easier to relate to algebraic cycles, than Hodge structures. Perhaps the point is that the Tate conjecture is related to finiteness problems in number theory, as discussed below. If we had a better qualitative understanding of number theory, then the Tate conjecture would be more accessible. Another point is that the Hodge conjecture predicts the Q-linear combinations of classes of subvarieties, whereas the Tate conjecture only predicts the $\mathbf{Q}_{l}$-span. So one could argue that the Tate conjecture is more complicated, because there is no way to start with a Galois-invariant class and come up with a single subvariety.

To spell out the relations between the Tate conjecture and finiteness problems, let $X$ be a smooth projective surface over a finite field $k$, and let $f$ be a morphism with connected fibers from $X$ onto a smooth projective curve $C$. Assume that the generic fiber $F$ of $f$, which is a curve over the function field $k(C)$, is smooth over $k(C)$. Let $J$ be the Jacobian of $F$; thus $J$ is an abelian variety over the global field $k(C)$. Then the following are equivalent [47, Proposition 5.1.2 and Theorem 6.3.1]:

- the Tate conjecture holds for divisors on $X$;

- the Brauer group of $X$ is finite;

- the Tate-Shafarevich group of $J$ is finite;

- the Birch-Swinnerton-Dyer conjecture holds for $J$. 
(This equivalence summarizes a lot of work by Tate, Artin, Milne, Kato, Trihan, and others.) These equivalent conjectures are very much open, despite a lot of progress.

A rough summary of the relations among these conjectures is as follows. Some of these conjectures will be discussed in section 2. Be aware that the different conjectures apply to varieties over different fields, and the implications have to be understood accordingly.

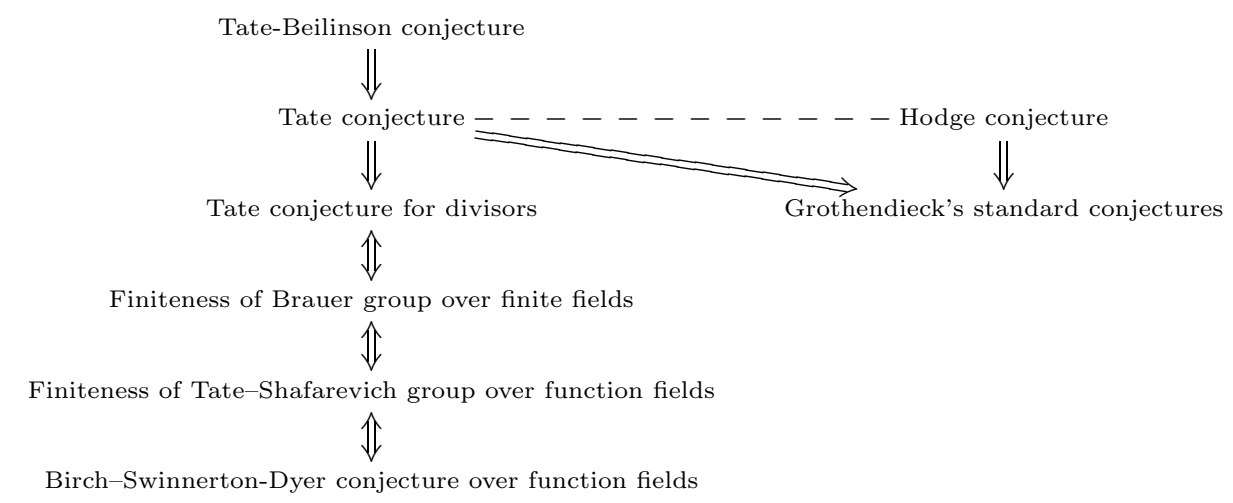

One direction not covered in this article is the proof of the Tate conjecture for many Shimura varieties. (Shimura varieties are locally symmetric varieties, with the model examples being the moduli spaces of abelian varieties.) One might speculate that Shimura varieties are the most important case to consider for the Tate conjecture. Indeed, the Langlands conjectures predict that the cohomology of any algebraic variety over a number field corresponds to an automorphic representation, with the simplest case being the cohomology of Shimura varieties. Some known cases are that the Tate conjecture for divisors holds for Hilbert modular surfaces and Picard modular surfaces [6, 14, 21, 33. A few cases of the Tate conjecture for higher-codimension cycles on Shimura varieties (such as Hilbert modular varieties) have also been proved [13, 36].

Typically, proofs of the Tate conjecture for Shimura varieties involve a particular class of subvarieties, Shimura subvarieties, but often they are not enough. Sometimes one can use the Lefschetz $(1,1)$-theorem to produce the missing divisors. Fundamentally, such approaches use that the theory of automorphic representations gives a particularly strong hold on the cohomology of Shimura varieties, both in terms of Hodge theory and in terms of Galois representations.

\section{THE BROADER CONTEXT: \\ VARIANTS OF THE TATE CONJECTURE}

Several attempts to strengthen the Tate conjecture aim for a more complete understanding of algebraic cycles. Some are false (but may be interesting in special cases), while others are still plausible. The variants we consider are the strong Tate conjecture; the Tate conjecture for singular or noncompact varieties; the Tate conjecture over the separable closure of a finitely generated field; the integral Tate conjecture; and the Tate-Beilinson conjecture. We also mention the consequences of the Tate conjecture for Grothendieck's "standard conjectures". 
The strong Tate conjecture is the combination of the Tate conjecture with the conjecture that, for a smooth projective variety over a finitely generated field $k$, the Galois-invariant subspace of $H^{2 a}\left(X, \mathbf{Q}_{l}(a)\right)$ is a summand, as a Galois representation. In several cases where the Tate conjecture has been proved (such as for divisors on abelian varieties), the arguments actually imply the strong Tate conjecture.

The strong Tate conjecture would imply most of Grothendieck's standard conjectures (except for the Hodge standard conjecture in positive characteristic) 2, section 7.3]. For example, one of the standard conjectures says that an algebraic cycle with nonzero image in $\mathbf{Q}_{l}$-cohomology must have nonzero intersection number with some algebraic cycle of complementary dimension; that is, "numerical and homological equivalence of algebraic cycles coincide."

Jannsen observed that the Tate conjecture can be generalized to any algebraic variety $X$ (not necessarily smooth or compact) over a finitely generated field, using $l$-adic Borel-Moore homology in place of $l$-adic cohomology. Namely, every Galoisinvariant element of $H_{2 a}\left(X, \mathbf{Q}_{l}(a)\right)$ should be the class of an algebraic cycle with $\mathbf{Q}_{l}$ coefficients. In fact, this more general conjecture essentially follows from the usual Tate conjecture (the case where $X$ is smooth and projective). The deduction works whenever $X$ has a resolution of singularities with a smooth compactification [17, Theorem 7.10].

In another direction, the Tate conjecture for a smooth projective variety $X$ over a finitely generated field $k$ follows easily from the conjecture for the base change $X_{E}$, for any finite Galois extension field $E$. Indeed, assume the conjecture for $X_{E}$, and let $u$ be an element of $H^{2 a}\left(X, \mathbf{Q}_{l}(a)\right)$ fixed by $\operatorname{Gal}\left(k_{s} / k\right)$. Then $u$ is fixed by the open subgroup $\operatorname{Gal}\left(k_{s} / E\right)$, and so it is the class of some algebraic cycle $\alpha$ on $X_{E}$. Since $\pi: X_{E} \rightarrow X$ is a finite morphism of schemes, the pushforward $\pi_{*}(\alpha)$ is an algebraic cycle on $X$. Using that $u$ is fixed by the whole group $\operatorname{Gal}\left(k_{s} / k\right)$, one checks that the cohomology class of $\pi_{*}(\alpha)$ is equal to $[E: k]$ times $u$, and so $u$ is algebraic.

As a result, one can always make finite extensions of the base field in trying to prove the Tate conjecture. Another version of the Tate conjecture is that for a variety $X$ over a finitely generated field $k$, every class in $H^{2 a}\left(X, \mathbf{Q}_{l}(i)\right)$ fixed by some open subgroup of $\operatorname{Gal}\left(k_{s} / k\right)$ is represented by an algebraic cycle over some finite extension of $k$. This is equivalent to the usual statement of the Tate conjecture over every finite extension field of $k$.

The integral Tate conjecture is the analogous statement about algebraic cycles with $\mathbf{Z}_{l}$ rather than $\mathbf{Q}_{l}$ coefficients. Most versions of the integral Tate conjecture are false in general, as discussed in [46, introduction]. On the other hand, for varieties over a finite field $k$, the usual Tate conjecture for divisors would imply the integral Tate conjecture for divisors [43, equation 5.10]. Also, for varieties over the algebraic closure of a finite field, the usual Tate conjecture for divisors on surfaces would imply the integral Tate conjecture for 1-cycles on varieties of any dimension, by Schoen [39, Theorem 0.5].

An important consequence of the strong Tate conjecture over finite fields would be a complete description of the category of Grothendieck motives over a finite field $k$. The result would be that every motive over $k$ is a Tate twist of a summand of the motive of an abelian variety tensored with an Artin motive [30, Remark 2.7]. (That is, roughly speaking, the cohomology of every variety over a finite field should be 
contained in the cohomology of some abelian variety, with the identification made by some algebraic correspondence between the two varieties.) This is far from true over other fields.

Finally, for varieties over finite fields, there is an important strengthening of the Tate conjecture, the Tate-Beilinson conjecture. Namely, let $X$ be a smooth projective variety over a finite field $k$. Write $C H^{a}(X)$ for the Chow group of codimension$a$ algebraic cycles modulo rational equivalence. Then Beilinson conjectured in the mid-1980s that the cycle map to $l$-adic cohomology,

$$
C H^{a}(X) \otimes \mathbf{Q} \rightarrow H^{2 a}\left(X, \mathbf{Q}_{l}(a)\right),
$$

is injective [19, Conjecture 50]. Combined with the Tate conjecture, this would give an optimally close connection between algebraic cycles and cohomology, for varieties over finite fields.

This is an extremely strong conjecture. For varieties $X$ over other fields, Chow groups can be big in various ways, and they do not inject into $l$-adic cohomology. For example, $C H^{1}$ of an elliptic curve $X$ is the direct sum of $\mathbf{Z}$ with the MordellWeil group $X(k)$, where $X(k)$ maps to zero in $H^{2}\left(X, \mathbf{Q}_{l}(i)\right) \cong \mathbf{Q}_{l}$. Here $X(k)$ can be uncountable for $k=\mathbf{C}$, and it can be infinite for $k=\mathbf{Q}$. On the other hand, $X(k)$ is finite for a finite field $k$. More generally, the group of $k$-points on an abelian variety over a finite field $k$ is finite, which implies Beilinson's conjecture for codimension-1 cycles. Beyond that case, evidence for Beilinson's conjecture is limited, but it holds in some interesting cases by Soulé [40] and Kahn [18.

\section{First RESUlts OVER FINITE FIELDS:}

The TATE CONJECTURE FOR DIVISORS ON ABELIAN VARIETIES

Probably the most important known case of the Tate conjecture is for divisors (codimension-1 cycles) on abelian varieties. In this section, we describe Tate's proof of this result for abelian varieties over finite fields [44. The argument shows the possibility of bringing geometric finiteness arguments to bear on the Tate conjecture, and it has helped to inspire many of the later developments. We present a simplification of Tate's original proof, using Zarhin's trick, which also plays a role in the recent proof for K3 surfaces (section [5).

For higher-codimension cycles on an abelian variety over a finite field, the Tate conjecture remains a tantalizing challenge. See Milne's survey of the Tate conjecture over finite fields for some known results [31.

Before sketching Tate's proof of the Tate conjecture for divisors on an abelian variety over a finite field, we introduce an equivalent formulation, very useful in itself. Namely, let $A$ and $B$ be abelian varieties over a finite field $k$. Let $l$ be a prime number invertible in $k$. For abelian varieties over any field, there is a natural homomorphism

$$
\operatorname{Hom}_{k}(A, B) \otimes \mathbf{Z}_{l} \rightarrow \operatorname{Hom}_{\mathrm{Gal}\left(k_{s} / k\right)}\left(H_{1}\left(A, \mathbf{Z}_{l}\right), H_{1}\left(B, \mathbf{Z}_{l}\right)\right),
$$

which is injective. Tate's theorem is that for $k$ finite, this homomorphism is actually bijective. This follows from the Tate conjecture for divisors on $A \times B$, and conversely this statement in the case $A=B$ implies the Tate conjecture for divisors on $A$. 
These relations between divisors and homomorphisms of abelian varieties can be deduced from the isomorphism

$$
N S(A \times B) \cong N S(A) \oplus N S(B) \oplus \operatorname{Hom}_{k}(A, \widehat{B})
$$

for abelian varieties over any field, where $N S$ denotes the Néron-Severi group (the image of the group of divisors with integer coefficients in $H^{2}$ ) and $\widehat{B}$ is the dual abelian variety of $B$. (To see why divisors have anything to do with homomorphisms of abelian varieties, note that a divisor $D$ on an abelian variety $X$ determines a homomorphism from $X$ to the dual abelian variety $\widehat{X}=\operatorname{Pic}^{0}(X)$, by

$$
x \mapsto x^{*}(D)-D,
$$

where $x^{*}(D)$ denotes the translate of $D$ by the point $x$ in $D$.)

A line bundle $L$ on a projective variety $X$ is ample if some positive multiple of $L$ has enough global sections to give an embedding of $X$ into some projective space. A polarization of an abelian variety $X$ is a homomorphism $f: X \rightarrow \widehat{X}$ that comes from some ample line bundle $L$ on $X$. (Knowing $f$ amounts to remembering only the class of $L$ in the Néron-Severi group $N S(X)$.) For $L$ ample, $f$ is an isogeny, meaning that it is surjective and has finite kernel. The degree of the polarization means the order of the kernel. Equivalently, for $g=\operatorname{dim}(X)$, the degree of the polarization is the intersection number $(1 / g !) c_{1}(L)^{g}$. A principal polarization means a polarization of degree 1.

We now sketch the proof of the Tate conjecture for divisors on an abelian variety over a finite field. We use a simplification of the proof based on Zarhin's trick; a survey of this approach is 25]. Zarhin's trick says that if $A$ is an abelian variety over an arbitrary field, then $(A \times \widehat{A})^{4}$ has a principal polarization 29, Remark 16.12]. The proof uses Lagrange's theorem that every positive integer is a sum of four squares. There is a large group of line bundles on $(A \times \widehat{A})^{4}$, and Lagrange's theorem makes it possible to find a polarization of degree 1.

This is important because the abelian varieties of a fixed dimension do not form a bounded family; that is, they are not parametrized by the points of finitely many algebraic varieties. Instead, there is a different moduli space for abelian varieties with a polarization of each degree $d \geq 1$. Nonetheless, Zarhin's trick shows that every abelian variety of given dimension $g$ can be embedded (possibly as a very highdegree abelian subvariety) in a principally polarized abelian variety of dimension $8 g$, and the latter varieties form a bounded family.

The endomorphism algebra of an abelian variety over any field is an algebra of finite rank over $\mathbf{Z}$, and tensoring with $\mathbf{Q}$ yields a semisimple algebra. Using the structure of such algebras, one finds that a given abelian variety has only finitely many summands, up to isomorphism [29, Theorem 18.7].

As a result, Zarhin's trick has a strong consequence for abelian varieties over a finite field $k$. By the existence of moduli spaces, we know that there are only finitely many isomorphism classes of abelian varieties of given dimension $g$ over $k$ with a polarization of given degree $d \geq 1$. In particular, there are only finitely many principally polarized abelian varieties of dimension $8 g$ over $k$. By Zarhin's trick, it follows that every abelian variety of dimension $g$ over the finite field $k$ is a summand of one of these finitely many abelian varieties. We conclude that there are only finitely many isomorphism classes of abelian varieties over $k$ of dimension $g$. Here the degree of the polarization is not specified. 
The Tate conjecture for divisors on abelian varieties over finite fields $k$ is deduced as follows. Let $A$ be an abelian variety over $k$. The main step is to show the following:

$(*)$ For every Galois-invariant submodule $W$ of $V_{l}:=H_{1}\left(A, \mathbf{Q}_{l}\right)$, there is an element $u$ of $\operatorname{End}(A) \otimes \mathbf{Q}_{l}$ such that $u\left(V_{l}\right)=W$.

This result (applied to $A \times A$ ) implies the formulation of the Tate conjecture in terms of endomorphisms: for a Galois-equivariant endomorphism $f$ of $V_{l}(A)$, the graph of $f$ is a Galois-invariant submodule of $V_{l}(A \times A)$ and $(*)$ can be applied to produce an element of $\operatorname{End}(A) \otimes \mathbf{Q}_{l}$ whose cohomology class is $f$, as we want.

To prove $(*)$ : For each natural number $n$, consider the subgroup

$$
X_{n}=\left(T_{l}(A) \cap W\right)+l^{n} T_{l}(A)
$$

of the Tate module. Since this is a Galois-invariant open subgroup, it corresponds to a finite subgroup $S_{n}$ (of order a power of $l$ ) in the dual abelian variety $\widehat{A}$ defined over $k$. This subgroup therefore determines an isogeny $\widehat{A} \rightarrow \widehat{A} / S_{n}$; dualizing gives an isogeny $f_{n}: B_{n} \rightarrow A$, with $f_{n}\left(T_{l}\left(B_{n}\right)\right)=X_{n}$.

Thus we have an infinite sequence of $g$-dimensional abelian varieties $B_{n}$ over the finite field $k$. By the argument above, there are only finitely many isomorphism classes of such abelian varieties. Thus there is a natural number $r$ such that $B_{r}$ is isomorphic to $B_{n}$ for infinitely many integers $n$. Choosing such isomorphisms, the isogenies $f_{n}$ can be viewed as endomorphisms of $B_{r}$. This sequence of endomorphisms must have a limit in the compact group $\operatorname{End}\left(B_{r}\right) \otimes \mathbf{Z}_{l}$. Finally, since $B_{r}$ is isogenous to the original abelian variety $A$, we have produced an element of $\operatorname{End}(A) \otimes \mathbf{Q}_{l}$ with the desired image. That completes the proof.

\section{MAIN TOOL:}

\section{The Kuga-Satake CORREspondence}

In this section, we discuss several results on the Tate conjecture for divisors on varieties over a number field, including recent progress by Moonen. The key tools in this context are Faltings's theorem and the Kuga-Satake correspondence, which relates $H^{2}$ of certain varieties with $H^{2}$ of an abelian variety.

The Tate conjecture is known for divisors on an abelian variety over a number field, by Faltings. The background is the work on the Mordell conjecture by Parshin, Arakelov, Zarhin, and others, leading to the 1983 proof by Faltings of the Mordell conjecture: every curve of genus at least 2 over a number field has only finitely many rational points [12]. As part of the proof, Faltings proved the Tate conjecture for divisors on an abelian variety over a number field. (A great introduction to Faltings's proof is Mazur's Bulletin of the AMS paper [28]. There are also booklength expositions of the proof [10].) Using the results of Tate and Faltings, Zarhin deduced the Tate conjecture for divisors on an abelian variety over any finitely generated field.

Thanks to Faltings's theorem, more is now known about the Tate conjecture over number fields than in the apparently simpler case of finite fields, notably Theorem 4.1 below.

The Tate conjecture for divisors on a variety $X$ over a number field is trivial when the Hodge number $h^{2,0}(X)=\operatorname{dim} H^{0}\left(X, \Omega^{2}\right)$ is equal to zero, since then the Lefschetz $(1,1)$-theorem gives that all of $H^{2}\left(X_{\mathbf{C}}, \mathbf{Q}\right)$ is spanned by divisors. The case where $h^{2,0}$ is equal to 1 should also be easier than the general case. Namely, the 
Kuga-Satake correspondence says that a polarizable Hodge structure with Hodge numbers $(1, m, 1)$ is a summand of $H^{2}$ of some abelian variety (whereas this fails in general when the outer Hodge number is greater than 1) [22, [16, Chapter 4]. Thus one may hope to prove the Tate conjecture on divisors for varieties $X$ with $h^{2,0}=1$ by reducing to Faltings's theorem on abelian varieties.

This is not automatic, because we do not know that the Kuga-Satake construction is realized by an algebraic correspondence. Nonetheless, there is a long tradition of combining the Kuga-Satake conjecture with arguments on monodromy to prove the conclusions we want. In this way, Deligne proved the Weil conjectures for K3 surfaces over finite fields. Even though he later proved the Weil conjectures for all varieties by a different method, the proof for K3 surfaces has been influential [11]. Tankeev also built on the Kuga-Satake construction to prove the Tate conjecture for K3 surfaces over number fields [41. Building on these ideas, André proved the Tate conjecture for divisors on irreducible hyperkähler varieties over number fields [1]. (For the purpose here, a hyperkähler variety is a simply connected smooth projective variety with $h^{2,0}(X)=\operatorname{dim} H^{0}\left(X, \Omega^{2}\right)$ equal to 1 such that a nonzero holomorphic 2-form on $X$ is nondegenerate.) In particular, although we do not know that the Kuga-Satake construction is given by an algebraic correspondence, it preserves Tate classes in this situation, as well as Hodge classes.

To sketch André's argument: given a Tate class $u$ in $H^{2}$ of a hyperkähler variety $X$ over a number field, the Kuga-Satake correspondence gives a Tate class in $H^{2}$ of some abelian variety. By Faltings's theorem, this is the class of a divisor with $\mathbf{Q}_{l}$ coefficients on the abelian variety, and so it is a $\mathbf{Q}_{l}$-linear combination of Hodge classes. Finally, the Kuga-Satake correspondence in reverse says that these Hodge classes correspond to Hodge classes on the hyperkähler variety $X$, and so they are algebraic by the Lefschetz $(1,1)$-theorem.

Recently, Moonen used the Kuga-Satake correspondence to prove the Tate conjecture for divisors on a larger class of varieties with $h^{2,0}=1$ :

Theorem 4.1. Let $X$ be a smooth proper variety with $h^{2,0}(X)=1$ over a finitely generated field $k$ of characteristic 0 . Assume that there is an embedding of $k$ into $\mathbf{C}$ such that $X_{\mathbf{C}}$ has a deformation (over some smooth base variety) in which the Hodge structure on $H^{2}\left(X_{\mathbf{C}}, \mathbf{Q}\right)$ varies nontrivially. Then the Tate conjecture for divisors holds for $X$.

The assumption that the Hodge structure varies nontrivially holds for most known surfaces with $h^{2,0}=1$, including many of general type [32, Theorem 9.4].

\section{Further RESUlts OVER Finite Fields: \\ The TATE CONJECTURE FOR K3 SURFACES}

We now discuss the work on the Tate conjecture for K3 surfaces over finite fields, including the recent complete proofs. This effort overcame significant new difficulties compared to the Tate conjecture for K3 surfaces over number fields (discussed in section 4). (On the other hand, the whole proof for K3 surfaces over number fields is probably still longer, in the sense that it relies on Faltings's theorem.)

The hard case is that of supersingular K3 surfaces, a special but fairly large class of K3 surfaces in positive characteristic, with very different properties from K3 surfaces in characteristic 0. In particular, for a supersingular K3 surface, the 
Tate conjecture predicts that the whole group $H^{2}$ (of dimension 22) is spanned by divisors. For K3 surfaces in characteristic 0, the Picard number (the dimension of the subspace of $H^{2}$ spanned by divisors) is always at most 20 .

The proofs we consider have a strong analogy with Tate's proof in the case of divisors on abelian varieties (section 3). Namely, one argues that if the Tate conjecture for some K3 surface over a finite field $k$ were false, then one could construct an infinite sequence of related K3 surfaces with the discriminant of the Néron-Severi lattice tending to infinity. But one argues that these related varieties have bounded degree, using a version of Zarhin's trick, and hence that there are only finitely many isomorphism classes of K3 surfaces that can arise this way over the finite field $k$. This contradicts the statement about the discriminant, thus implying the Tate conjecture for the original K3 surface.

The Tate conjecture was proved by Artin and Swinnerton-Dyer for K3 surfaces with an elliptic fibration [3, and by Rudakov, Shafarevich, and Zink for K3 surfaces of degree 2 [38, Theorem 4]. For non-supersingular K3 surfaces in characteristic $p>5$, the Tate conjecture was proved by Nygaard and Ogus in 1985 [34. Finally, the supersingular case was proved in a series of papers starting in 2012 by Charles, Kim, Madapusi Pera, and Maulik [7, 20, 26, 27]. The case of characteristic 2 was proved only in the last of these papers, by Kim and Madapusi Pera.

5.1. Definitions. By definition, a $K 3$ surface $X$ over a field is a smooth projective surface with trivial canonical bundle $K_{X}:=\Omega_{X}^{2}$ and Betti number $b_{1}(X)$ equal to zero [16]. Using the Riemann-Roch theorem, it follows that $X$ has second Betti number equal to 22, with Hodge diamond

$\begin{array}{cccccc} & & 1 & & \\ & & 0 & & 0 & \\ & & 20 & & 1 \\ & 0 & & 0 & \\ & & & 1 . & & \end{array}$

Since $X$ is projective, it has an ample line bundle $L$, and the degree of $(X, L)$ means the intersection number $c_{1}(L)^{2}$; this is a positive even number.

In general, a smooth projective variety $X$ over a field $k$ of characteristic $p>0$ is called supersingular if the absolute Frobenius eigenvalues on the cohomology $H^{i}(X)$ all have $p$-adic absolute value equal to $p^{i / 2}$. (In other words, the slopes of Frobenius on crystalline cohomology $H^{i}(X)$ are all equal to $i / 2$. This is the usual definition of a supersingular elliptic curve or abelian variety.) For a finite field $k$, the definition of "supersingular" is equivalent to saying that all eigenvalues of Frobenius on $H^{i}\left(X, \mathbf{Q}_{l}\right)$ are equal to $q^{i / 2}$ times a root of unity, where $q$ is the order of $k$. As a result, the Tate conjecture would predict that all the even-dimensional cohomology of a supersingular variety over $\overline{\mathbf{F}}_{p}$ is spanned by algebraic cycles. Since every K3 surface has second Betti number 22, the problem is to show that a supersingular K3 surface has Picard number 22. This never happens for K3 surfaces in characteristic 0 , where the Hodge numbers $(1,20,1)$ of $H^{2}(X)$ imply that the Picard number of $X$ is at most 20 .

The proof of the Tate conjecture for supersingular K3 surfaces has inspired other striking progress on the geometry of these surfaces. In particular, in characteristic $p \geq 5$, Liedtke proved Artin's conjecture that every supersingular K3 surface $X$ is unirational [24]. (That is, there is a dominant rational map $\mathbf{P}^{2}-\rightarrow X$.) Again, this 
is something that can never happen in characteristic 0: a smooth projective variety in characteristic 0 with nonzero regular differential forms of positive degree, such as a K3 surface, cannot be unirational.

Supersingular K3 surfaces turn out to form a large subclass of all K3 surfaces in characteristic $p>0$. Namely, they form a 9-dimensional family inside the 19dimensional moduli space of all projective K3 surfaces of given degree 35, Theorem 15]. By contrast, the moduli spaces of non-supersingular K3 surfaces with Picard number $\rho$ have dimension $20-\rho$.

5.2. Elliptic K3 surfaces (following Artin and Swinnerton-Dyer). Artin and Swinnerton-Dyer's proof for an elliptic K3 surface $X \rightarrow \mathbf{P}^{1}$ uses the equivalence between the Tate conjecture for $X$ and the finiteness of the Tate-Shafarevich group $\amalg$ for the bundle of Jacobians $A$ of $X$ over $\mathbf{P}^{1}$. More precisely, if the Tate conjecture fails, then for any prime $l \neq p$, there is a sequence $\beta_{n}, n \geq 0$, of elements of $H^{1}\left(\mathbf{P}_{k}^{1}, A\right)$ with $l \beta_{n+1}=\beta_{n}$ and $\beta_{0} \neq 0$. In geometric terms, these cohomology classes represent a sequence of principal homogeneous spaces for $A$ over $\mathbf{P}^{1}$, which we compactify to a sequence of projective surfaces:

$$
\cdots \rightarrow X_{2} \rightarrow X_{1} \rightarrow X_{0}
$$

These are all K3 surfaces over $k$. The key step of Artin and Swinnerton-Dyer's proof uses the Riemann-Roch theorem to show that all these surfaces $X_{n}$ have nef and big line bundles $L_{n}$ of bounded degree, independent of $n$. (By definition, a line bundle on a projective variety $X$ is nef if it has nonnegative degree on every curve. A line bundle is big if some positive multiple of $L$ has enough global sections to give a birational embedding of $X$ into some projective space.)

Using that each surface $X_{n}$ is a K3 surface, it is known that 2 times the line bundle $L_{n}$ gives a birational map from $X_{n}$ into projective space. Since $\left(X_{n}, L_{n}\right)$ has bounded degree, the image is a projective variety with degree bounded independent of $n$. But everything is defined over the finite field $k$, and so there are only finitely many varieties of given dimension and degree in projective space over $k$. So the varieties $X_{n}$ fall into only finitely many birational equivalence classes. Finally, K3 surfaces are minimal (that is, they are smooth projective varieties with nef canonical bundle), and two minimal surfaces that are birational are isomorphic. Therefore, the varieties $X_{n}$ fall into only finitely many isomorphism classes. In particular, there is an infinite set $I$ of natural numbers such that all the surfaces $X_{n}$ for $n \in I$ are isomorphic.

Given that, the end of Artin and Swinnerton-Dyer's proof is as follows. We have infinitely many elliptic fibrations on a single K3 surface, with higher and higher indices (the gcd of the degrees of all multisections). This is impossible, since a fairly elementary argument shows that on a given $\mathrm{K} 3$ surface $Y$, there is an integer $N$ such that every pencil of elliptic curves on $Y$ has a multisection of degree $\leq N$. (The proof uses that the class of an elliptic curve is a primitive class in the NéronSeveri group $N$ with self-intersection zero, and that such elements fall into only finitely many orbits under the orthogonal group of $N$.)

5.3. Non-supersingular K3 surfaces (following Nygaard and Ogus). Nygaard and Ogus's proof of the Tate conjecture for non-supersingular K3 surfaces $X$ over finite fields uses a different approach: reduction to the Hodge conjecture for divisors. Namely, using the well-behaved deformation theory of K3 surfaces, they construct a lifting $Y$ of $X$ to $\mathbf{C}$ such that the action of the Frobenius map on the 
crystalline cohomology of $X$ corresponds, via the canonical isomorphism between crystalline and de Rham cohomology, to an endomorphism of the Hodge structure of $Y$. They call this a "quasi-canonical lifting", by analogy with the Serre-Tate canonical lifting of an ordinary abelian variety over a finite field. That property implies that we get as many 1 -cycles as we need on $X$ by reduction $\bmod p$ of 1 -cycles on $Y$, using the Hodge conjecture for divisors on $Y$.

This argument does not have a direct analog for supersingular K3 surfaces $X$. Indeed, the goal is to show that $X$ has Picard number 22, but any lifting of $X$ to characteristic 0 has Picard number at most 20. So $X$ cannot have a quasi-canonical lifting in the same sense.

To prove the Tate conjecture for supersingular K3 surfaces, Maulik, Charles, and Madapusi Pera all (in various levels of generality) extended the Kuga-Satake construction to mixed characteristic. They also proved versions of the Torelli theorem for K3 surfaces in mixed characteristic. These arguments used the steady advances in $p$-adic Hodge theory over several decades, including work of Bloch, Kato, Fontaine, Kisin, and others [4, section 1.6].

5.4. Charles's proof. To discuss the proof of the Tate conjecture for K3 surfaces in more detail, we will describe a second-generation proof by Charles [8. This proof still uses the Kuga-Satake correspondence, but to a lesser extent than in the earlier proofs. The new proof has the appeal that its main focus is an analog of Zarhin's trick for K3 surfaces (rather than abelian varieties).

Charles's version of Zarhin's trick for K3 surfaces $X$ uses a moduli space of sheaves on $X$ in place of the abelian variety $(A \times \widehat{A})^{4}$. Such moduli spaces are hyperkähler varieties, and we need to state a basic property of such varieties $Y$. Namely, there is a natural quadratic form $q$ on the cohomology $H^{2}(Y)$, the Beauville-Bogomolov quadratic form. This form is characterized by the property that there is a positive constant $a$ such that the intersection number $u^{2 n}$ is equal $a q(u)^{n}$ for all $u$ in $H^{2}(Y)$, where $2 n$ is the dimension of $Y$. More precisely, this determines the quadratic form $q$ on $H^{2}$ up to a scalar, which one can fix by requiring that $q$ makes the Néron-Severi group $N S(Y)$ into a primitive integral lattice.

As with abelian varieties, the difficulty is that projective K3 surfaces do not form a bounded family. Rather, there is a (19-dimensional) moduli space of K3 surfaces of degree $d$ for each even integer $d \geq 2$. However, Charles shows that for any field $k$, there is a positive integer $r$ and there are infinitely many positive integers $m$ (given by a congruence condition) such that for every K3 surface of degree $2 m$ over $k$, there is a smooth, 4-dimensional, projective moduli space $M$ of stable sheaves on $X$ and a line bundle $L$ on $M$ such that $q(L)=r$ and $L$ is big [8, Theorem 1.1].

Thus the moduli space $(M, L)$ has bounded degree, even though it arises from a K3 surface which may have arbitrarily large degree. One would like to conclude that every such moduli space $M$ is at least birational to a subvariety of bounded degree in a fixed projective space.

Such a statement may eventually be provable by geometric methods, as can be done now in characteristic 0. For now, in positive characteristic, Charles is forced to prove a result in this direction using the Kuga-Satake construction, reducing to the case of abelian varieties. The result is that, by the boundedness of $q(L)$, there are only finitely many possibilities for the Néron-Severi lattice of $M[8$, Proposition $2.6]$. 
Applying this result over a finite field $k$, Charles deduces that there are only finitely many isomorphism classes of K3 surfaces over $k$, with some restrictions on the degree (but a priori allowing arbitrarily high degrees) [8, Proposition 3.17].

The final step of the proof uses moduli spaces of twisted sheaves on a K3 surface, following the approach advocated by Lieblich, Maulik, and Snowden [23]. (Their argument generalizes the tower of elliptic surfaces in Artin and Swinnerton-Dyer's proof.) Namely, Lieblich, Maulik, and Snowden showed, for a finite field $k$ of characteristic $p>5$, that the Tate conjecture holds for K3 surfaces over the algebraic closure of $k$ if and only if there are only finitely many isomorphism classes of K3 surfaces over each finite extension of $k$. At this point in the proof, Charles has proved a weaker version of the second statement (with some restrictions on the degree), but this is enough to yield the Tate conjecture for K3 surfaces 8 , section 4].

\section{Conclusion}

Where can we expect further advances on the Tate conjecture? One big step, extending the proof for K3 surfaces over finite fields, would be to prove the Tate conjecture for divisors on more or less arbitrary varieties $X$ over finite fields such that the Hodge number $h^{2,0}(X)$ is equal to 1 . This would be analogous to Moonen's result over number fields (Theorem 4.1). The point is that the assumption on $h^{2,0}$ might allow a version of the Kuga-Satake correspondence to reduce the problem to the known case of divisors on abelian varieties.

For varieties with $h^{2,0}>1$, it seems that very different methods will be needed. For the corresponding complex varieties $X$, the Hodge structure on $H^{2}(X)$ usually cannot be imbedded into $H^{2}$ of an abelian variety. Perhaps Zarhin's trick can be extended to new cases. Also, it seems likely that the known relations between the Hodge conjecture and the Tate conjecture can be pushed further. Since the Hodge conjecture for divisors is known, such relations would be important for the Tate conjecture.

Finally, it is possible that progress will come through advances on the BirchSwinnerton-Dyer conjecture. Yun and Zhang's extension of the Gross-Zagier formula to higher derivatives of $L$-functions (in the function field case) is a very promising step [48.

\section{ACKNOWLEDGMENTS}

The author thanks François Charles, Martin Gallauer, Diana Gillooly, Chandrashekhar Khare, and Romyar Sharifi for their suggestions.

\section{AbOut THE AUTHOR}

Burt Totaro is professor of mathematics at University of California, Los Angeles, and is a Fellow of the Royal Society.

\section{REFERENCES}

[1] Yves André, On the Shafarevich and Tate conjectures for hyper-Kähler varieties, Math. Ann. 305 (1996), no. 2, 205-248, DOI 10.1007/BF01444219. MR.1391213

[2] Yves André, Une introduction aux motifs (motifs purs, motifs mixtes, périodes) (French, with English and French summaries), Panoramas et Synthèses [Panoramas and Syntheses], vol. 17, Société Mathématique de France, Paris, 2004. MR2115000 
[3] M. Artin and H. P. F. Swinnerton-Dyer, The Shafarevich-Tate conjecture for pencils of elliptic curves on K3 surfaces, Invent. Math. 20 (1973), 249-266, DOI 10.1007/BF01394097. MR.0417182

[4] Olivier Benoist, Construction de courbes sur les surfaces K3 (d'après Bogomolov-HassettTschinkel, Charles, Li-Liedtke, Madapusi Pera, Maulik,...) (French, with French summary), Astérisque 367-368 (2015), Exp. No. 1081, viii, 219-253. MR3363592

[5] B. J. Birch and H. P. F. Swinnerton-Dyer, Notes on elliptic curves. II, J. Reine Angew. Math. 218 (1965), 79-108, DOI 10.1515/crll.1965.218.79. MR0179168

[6] Don Blasius and Jonathan D. Rogawski, Tate classes and arithmetic quotients of the twoball, The zeta functions of Picard modular surfaces, Univ. Montréal, Montreal, QC, 1992, pp. 421-444. MR.1155236

[7] François Charles, The Tate conjecture for K3 surfaces over finite fields, Invent. Math. 194 (2013), no. 1, 119-145, DOI 10.1007/s00222-012-0443-y. MR3103257

[8] François Charles, Birational boundedness for holomorphic symplectic varieties, Zarhin's trick for K3 surfaces, and the Tate conjecture, Ann. of Math. (2) 184 (2016), no. 2, 487-526, DOI 10.4007/annals.2016.184.2.4. MR 3548531

[9] P. Colmez and J.-P. Serre, editors, Correspondance Serre-Tate, Volumes I and II (French). Documents Mathématiques (Paris) 13, 14. Societé Mathématique de France, Paris, 2015. MR3379329 MR3379330

[10] Gary Cornell and Joseph H. Silverman (eds.), Arithmetic geometry, Springer-Verlag, New York, 1986. Papers from the conference held at the University of Connecticut, Storrs, Connecticut, July 30-August 10, 1984. MR861969

[11] Pierre Deligne, La conjecture de Weil pour les surfaces K3 (French), Invent. Math. 15 (1972), 206-226, DOI 10.1007/BF01404126. MR0296076

[12] G. Faltings, Endlichkeitssätze für abelsche Varietäten über Zahlkörpern (German), Invent. Math. 73 (1983), no. 3, 349-366, DOI 10.1007/BF01388432. MR718935

[13] Jayce R. Getz and Heekyoung Hahn, Algebraic cycles and Tate classes on Hilbert modular varieties, Int. J. Number Theory 10 (2014), no. 1, 161-176, DOI 10.1142/S1793042113500875. MR3189973

[14] G. Harder, R. P. Langlands, and M. Rapoport, Algebraische Zyklen auf Hilbert-BlumenthalFlächen (German), J. Reine Angew. Math. 366 (1986), 53-120. MR833013

[15] Daniel Huybrechts, Complex geometry: An introduction, Universitext, Springer-Verlag, Berlin, 2005. MR.2093043

[16] Daniel Huybrechts, Lectures on K3 surfaces, Cambridge Studies in Advanced Mathematics, vol. 158, Cambridge University Press, Cambridge, 2016. MR3586372

[17] Uwe Jannsen, Mixed motives and algebraic K-theory, Lecture Notes in Mathematics, vol. 1400, Springer-Verlag, Berlin, 1990. With appendices by S. Bloch and C. Schoen. MR 1043451

[18] Bruno Kahn, Équivalences rationnelle et numérique sur certaines variétés de type abélien sur un corps fini (French, with English and French summaries), Ann. Sci. École Norm. Sup. (4) 36 (2003), no. 6, 977-1002 (2004), DOI 10.1016/j.ansens.2003.02.002. MR2032532

[19] Bruno Kahn, Algebraic K-theory, algebraic cycles and arithmetic geometry, Handbook of K-theory. Vol. 1, 2, Springer, Berlin, 2005, pp. 351-428, DOI 10.1007/3-540-27855-9_9. MR2181827

[20] Wansu Kim and Keerthi Madapusi Pera, 2-adic integral canonical models and the Tate conjecture in characteristic 2. arXiv: 1512.02540

[21] Christoph Klingenberg, Die Tate-Vermutungen für Hilbert-Blumenthal-Flächen (German), Invent. Math. 89 (1987), no. 2, 291-317, DOI 10.1007/BF01389080. MR894381

[22] Michio Kuga and Ichirô Satake, Abelian varieties attached to polarized $K_{3}$-surfaces, Math. Ann. 169 (1967), 239-242, DOI 10.1007/BF01399540. MR0210717

[23] Max Lieblich, Davesh Maulik, and Andrew Snowden, Finiteness of K3 surfaces and the Tate conjecture (English, with English and French summaries), Ann. Sci. Éc. Norm. Supér. (4) 47 (2014), no. 2, 285-308, DOI 10.24033/asens.2215. MR3215924

[24] Christian Liedtke, Supersingular K3 surfaces are unirational, Invent. Math. 200 (2015), no. 3, 979-1014, DOI 10.1007/s00222-014-0547-7. MR3348142

[25] D. Lombardo. Conjecture de Tate sur les corps finis. https://www.math.u-psud.fr/ lombardo/Mordell/TateConjectureOverFiniteFields.pdf

[26] Keerthi Madapusi Pera, The Tate conjecture for K3 surfaces in odd characteristic, Invent. Math. 201 (2015), no. 2, 625-668, DOI 10.1007/s00222-014-0557-5. MR3370622 
[27] Davesh Maulik, Supersingular K3 surfaces for large primes, Duke Math. J. 163 (2014), no. 13, 2357-2425, DOI 10.1215/00127094-2804783. With an appendix by Andrew Snowden. MR.3265555

[28] Barry Mazur, Arithmetic on curves, Bull. Amer. Math. Soc. (N.S.) 14 (1986), no. 2, 207-259, DOI 10.1090/S0273-0979-1986-15430-3. MR828821

[29] J. S. Milne, Abelian varieties, Arithmetic geometry (Storrs, Conn., 1984), Springer, New York, 1986, pp. 103-150. MR861974

[30] J. S. Milne, Motives over finite fields, Motives (Seattle, WA, 1991), Proc. Sympos. Pure Math., vol. 55, Amer. Math. Soc., Providence, RI, 1994, pp. 401-459. MR.1265538

[31] J. S. Milne, The Tate conjecture over finite fields. arXiv:0709.3040

[32] Ben Moonen, On the Tate and Mumford-Tate conjectures in codimension 1 for varieties with $h^{2,0}=1$, Duke Math. J. 166 (2017), no. 4, 739-799, DOI 10.1215/00127094-3774386. MR3619305

[33] V. Kumar Murty and Dinakar Ramakrishnan, Period relations and the Tate conjecture for Hilbert modular surfaces, Invent. Math. 89 (1987), no. 2, 319-345, DOI 10.1007/BF01389081. MR894382

[34] Niels Nygaard and Arthur Ogus, Tate's conjecture for K3 surfaces of finite height, Ann. of Math. (2) 122 (1985), no. 3, 461-507, DOI 10.2307/1971327. MR819555

[35] Arthur Ogus, Singularities of the height strata in the moduli of K3 surfaces, Moduli of abelian varieties (Texel Island, 1999), Progr. Math., vol. 195, Birkhäuser, Basel, 2001, pp. 325-343. MR. 1827026

[36] Dinakar Ramakrishnan, Algebraic cycles on Hilbert modular fourfolds and poles of Lfunctions, Algebraic groups and arithmetic, Tata Inst. Fund. Res., Mumbai, 2004, pp. 221274. MR 2094113

[37] Wayne Raskind, A generalized Hodge-Tate conjecture for algebraic varieties with totally degenerate reduction over $p$-adic fields, Algebra and number theory, Hindustan Book Agency, Delhi, 2005, pp. 99-115. MR2193347

[38] A. N. Rudakov, I. R. Shafarevich, and T. Zink, The influence of height on degenerations of algebraic surfaces of type K3. Math. USSR Izv. 20 (1983), 119-135.

[39] Chad Schoen, An integral analog of the Tate conjecture for one-dimensional cycles on varieties over finite fields, Math. Ann. 311 (1998), no. 3, 493-500, DOI 10.1007/s002080050197. MR.1637931

[40] C. Soulé, Groupes de Chow et K-théorie de variétés sur un corps fini (French), Math. Ann. 268 (1984), no. 3, 317-345, DOI 10.1007/BF01457062. MR751733

[41] S. G. Tankeev, Surfaces of type K3 over number fields, and l-adic representations (Russian), Izv. Akad. Nauk SSSR Ser. Mat. 52 (1988), no. 6, 1252-1271, 1328; English transl., Math. USSR-Izv. 33 (1989), no. 3, 575-595. MR984218

[42] John T. Tate, Algebraic cycles and poles of zeta functions, Arithmetical Algebraic Geometry (Proc. Conf. Purdue Univ., 1963), Harper \& Row, New York, 1965, pp. 93-110. MR0225778

[43] John Tate, On the conjectures of Birch and Swinnerton-Dyer and a geometric analog, Dix exposés sur la cohomologie des schémas, Adv. Stud. Pure Math., vol. 3, North-Holland, Amsterdam, 1968, pp. 189-214. MR 3202555

[44] John Tate, Endomorphisms of abelian varieties over finite fields, Invent. Math. 2 (1966), 134-144, DOI 10.1007/BF01404549. MR0206004

[45] John Tate, Conjectures on algebraic cycles in l-adic cohomology, Motives (Seattle, WA, 1991), Proc. Sympos. Pure Math., vol. 55, Amer. Math. Soc., Providence, RI, 1994, pp. 71-83, DOI 10.1090/pspum/055.1/1265523. MR 1265523

[46] Burt Totaro, On the integral Hodge and Tate conjectures over a number field, Forum Math. Sigma 1 (2013), e4, 13 pp., DOI 10.1017/fms.2013.3. MR3143715

[47] Douglas Ulmer, Curves and Jacobians over function fields, Arithmetic geometry over global function fields, Adv. Courses Math. CRM Barcelona, Birkhäuser/Springer, Basel, 2014, pp. 283-337. MR3586808

[48] Zhiwei Yun and Wei Zhang. Shtukas and the Taylor expansion of L-functions. arXiv:1512. 02683

UClA Mathematics Department, Box 951555, Los Angeles, CA 90095-1555

E-mail address: totaro@math.ucla.edu 